\title{
360-Degree Distribution of Biofilm Quantity and Community in an Operational Unchlorinated Drinking Water Distribution Pipe
}

Gang Liu,* Ya Zhang, Xinlei Liu, Frederik Hammes, Wen-Tso Liu, Gertjan Medema, Peter Wessels, and Walter van der Meer

Cite This: Environ. Sci. Technol. 2020, 54, 5619-5628

Read Online

ACCESS

Llll Metrics \& More

Article Recommendations

Supporting Information

ABSTRACT: In the present study, triplicate rings of $360^{\circ}$ pipe surfaces of an operational drinking water distribution pipe were swabbed. Each ring was equally divided into 16 parts for swabbing. The collected swabs were grouped into 3 sections and compared with the biofilm samples sampled by sonication of specimens from the same pipe. The results showed that the biofilm is unevenly distributed over the 16 parts and the 3 sections of the pipe surface. Both the active biomass and the number of observed OTUs increased as the measurements proceeded from the top to the bottom of the pipe. The bacterial community was dominated in all sections by Proteobacteria. At the genus level, Nitrospira spp., Terrimonas spp., and Hyphomicrobium spp. were dominant in all

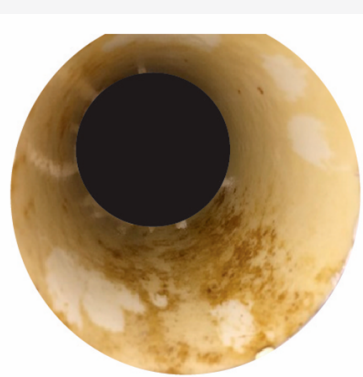

Picture of inner surface of a distribution pipe 360-degrees distribution of biofilm (PVC, 110mm)

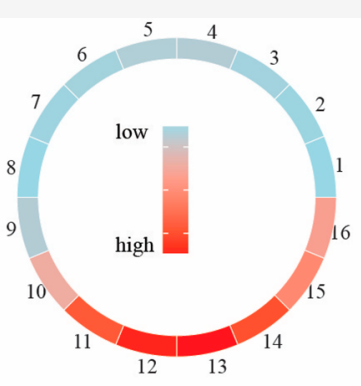

(ATP and OTUs) sections. Gaiella spp. and Vicinamibacter spp. dominated in S-I, Blastopirellula spp. and Pirellula spp. dominated in S-II, while Holophaga spp. and Phaeodactylibacter spp. dominated in S-III. When swabbing and pipe specimen sonication were compared, the results showed that the sampling strategy significantly influences the obtained biofilm bacterial community. A consistent multisectional swabbing strategy is proposed for future biofilm sampling; it involves collecting swabs from all sections and comparing the swabs from the same position/section across locations.

\section{INTRODUCTION}

The omnipresence of microbes in drinking water systems has been proven and acknowledged. ${ }^{1}$ In particular, the microbial accumulation on the pipe surface in drinking water distribution systems in the form of biofilm (0.1-4.1 $\mathrm{ng}$ ATP $\mathrm{cm}^{-2}$ ) has been widely documented, whether disinfectant residuals are applied or not. ${ }^{2}$ For drinking water biosafety, the importance of drinking water biofilms has been attributed to their higher resistance to disinfectants (if applicable), the related biocorrosion, the associated pathogenic bacteria, and their consistent release of biofilm bacteria into bulk water. ${ }^{3-5}$ Over the last decades, great efforts have been made to investigate (1) the physiochemical characteristics of biofilm, ${ }^{6}$ (2) the bacterial quantity and community of biofilm, ${ }^{2}$ (3) the origin and development of biofilm and the factors that impact biofilm development, ${ }^{7,8}$ and (4) the release of biofilm bacteria into bulk water and their influence on bulk water, ${ }^{5,9}$ (5) especially during disturbances like variations in supply water quality., 5,10

Due to the limited opportunities to assess real distribution networks, most of the biofilm studies have used surrogates, such as pilot-scale distribution systems and/or removable coupons temporarily inserted into simulated systems. ${ }^{11,12}$ Although valuable knowledge has been obtained in studies with such simulated distribution systems, there is a consensus that real distribution systems are difficult to simulate with pilot systems because of the real systems' complex of variable parameters (e.g., water-usage pattern, local hydraulics, pipe material, diameter, and length). ${ }^{13}$ Studies in real distribution systems are therefore seen as necessary and valuable.

In the limited number of studies on full-scale operational distribution systems, biofilm has been sampled by scraping surfaces of valves or water meters that have been in contact with drinking water, ${ }^{14,15}$ swabbing/scraping/brushing the pipe surface (considered to be a single biofilm sampling approach, hereinafter referred to as "swabbing"), ,6,16-18 or sonicating pieces of cut-out pipe (pipe specimen sonication). ${ }^{19}$ Sampling biofilm from valves and water meters in real distribution systems could present an alternative to studying pipe-wall biofilms from real distribution systems, but the valve and water meter biofilm can differ from the pipe-wall biofilm because of the influence on biofilm development of the different

Received: November 1, 2019

Revised: February 4, 2020

Accepted: April 7, 2020

Published: April 7, 2020

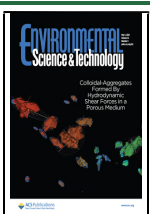




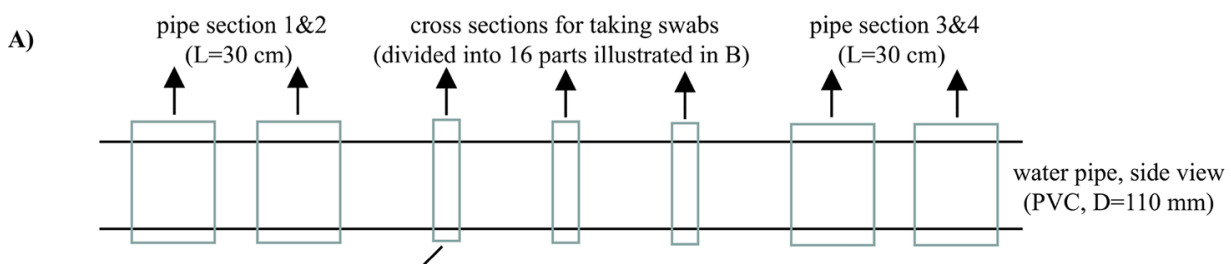

B)

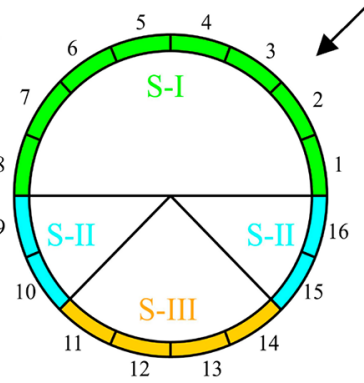

E)

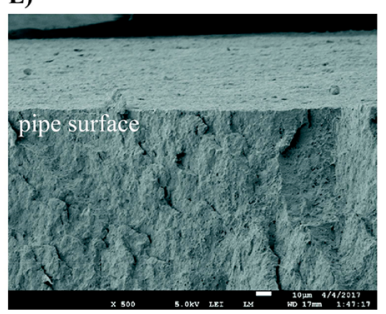

C)

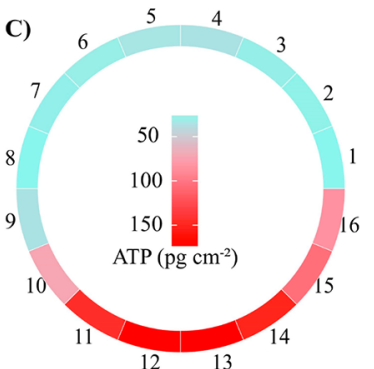

F)

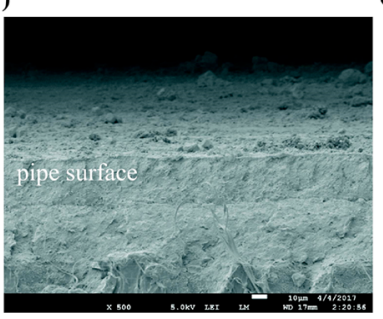

D)

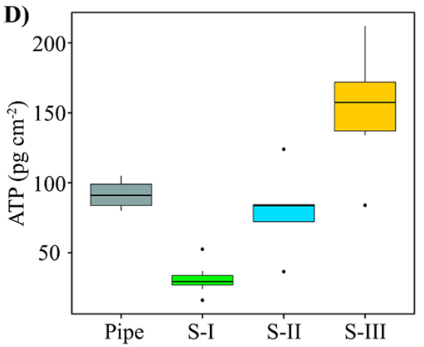

G)

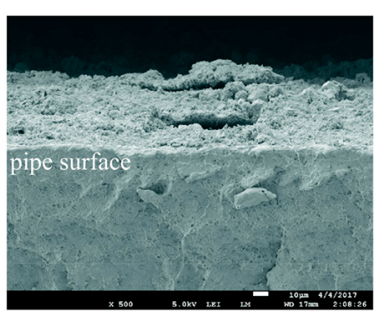

Figure 1. (A) Design of sampling. The length of harvested pipe is $150 \mathrm{~m}$. Two pieces of pipe sections were taken from each end of the pipe for sonication $(n=4$, pipe specimen, coded as Pipe). Three cross-sections were chosen for taking swabs, $8 / 16$ parts were swabbed randomly in each cross-section as calculated by R script. (B) The definition of 3 sections and 16 parts of pipe surface for swabbing. S-I: Section 1, the upper half divided into 8 parts (in green); S-II: Section 2, the upper half of the bottom section divided into 4 parts (in blue); S-III: Section 3, the surface area of pipe bottom divided into 4 parts (in brown). (C) The heatmap showing ATP by surface area over the 16 sampling areas within one crosssection. (D) Boxplot showing the active biomass as measured by ATP, results grouped by the pipes and swabbed positions (S-I, S-II, and S-III). (E-G) SEM pictures of pipe surfaces of different swab positions, the parts of 2, 15, and 13 defined (B) representing S-I (E), S-II (F), and S-III (G), respectively.

hydraulics and materials involved. ${ }^{20,21}$ For this reason, pipe specimen sonication and the swabbing of the pipe surface are better options for taking real distribution system biofilm samples. These are notoriously difficult to perform because both methods require the cutting of pipes (usually conducted during water-pipe renovation or repair). Pipe specimen sonication has been mostly applied to plastic water pipes with diameters no bigger than $110 \mathrm{~mm} .{ }^{8,19,22}$ However, swabbing is a more common practice than pipe specimen sonication, because of its easier handling and post-treatmentthis is especially the case when one considers the difficulties of processing pipe specimens when metal pipes of bigger diameters (e.g., $600 \mathrm{~mm}$ ) involved. ${ }^{23}$

However, for the swabbing approach, the position and surface area where the swabs are taken may introduce considerable biases if the biofilm is not evenly distributed over the water pipe surface. Yet, little attention has been given to the vertical distribution of biofilm on the pipe surface. Our earlier studies in drinking water distribution systems have demonstrated the considerable contribution of loose deposits at the bottom of distribution pipes to the total bacterial quantity and community composition, ${ }^{19}$ as well as the presence of hotspots for microbes and nutrient accumulation. ${ }^{22}$ It is conceivable that the variable availability of inorganic nutrients (e.g., $\mathrm{Fe}, \mathrm{Mn}$ ) and particulate organic carbon may give rise to variability in the quantity and community composition of biofilms, so that the biofilm will develop unevenly over the $360^{\circ}$ of a water pipe's cross-section. ${ }^{24,25}$ Additionally, the presence of gravity can have a substantial effect on biofilm formation and sloughing. ${ }^{26}$ This hypothesis is corroborated by a recent study that looked into the radial-spatial distribution of biofilms, which found that the middle of pipes have the highest abundance of biomass and the most diverse community. ${ }^{27}$ However, in that study only a single swab was taken from each of the three defined parts of pipe surface.

To understand the fundamental process of biofilm formation in water pipes and ensure the reliability of swabbing for biofilm sampling from operational distribution system pipes, the spatial distribution of biofilm over $360^{\circ}$ of the pipe surface is a critical research question that must imperatively be answered. The objective of this study was to investigate the $360^{\circ}$ spatial distribution of biofilm quantity and community composition on pipe surfaces in real distribution networks. In addition, biofilms were collected by both swabbing and cutting pipe specimens and treating them by sonication; the results obtained with the two methods were then compared.

\section{MATERIAL AND METHODS}

2.1. Water Supply System. At the treatment plant, the abstracted groundwater is treated by aeration, rapid sand filtration, softening, activated carbon filtration, and UV disinfection, before being pumped into the distribution system. In the treated water, the $\mathrm{Fe}, \mathrm{Mn}, \mathrm{Al}$, and $\mathrm{As}$ were under the detection limits, with about $23 \pm 1.2 \mathrm{mg} \mathrm{l}^{-1} \mathrm{Ca}$ and $8.0 \pm 2.3$ $\mathrm{ng} \mathrm{l}^{-1}$ ATP. The type of water main selected for this study is the one most widely used for drinking water distribution in 
The Netherlands, namely: PVC-U, with an external diameter of $110 \mathrm{~mm}$. The studied pipe was harvested from an operational distribution pipeline that has been in service for over 20 years $(L=150 \mathrm{~m})$.

2.2. Biofilm Sampling. As shown in Figure 1A, two pipe sections were cut out and ultrasonicated to collect biofilm from each end of the harvested pipe, while in the middle, three cross sections were selected for the swabbing of biofilm samples.

2.2.1. Pipe Cutting. The biofilm was sampled by cutting pipe sections as previously described. ${ }^{19}$ To maximize the environment's sterility when cutting the pipe, the following steps were taken: (1) after the hole was dug, the pipe was protected against possible contamination from the surrounding soil with a precleaned plastic sheet (Figure S1-A of the Supporting Information, SI); (2) the sheet was disinfected again with chlorine spray before the pipe was cut; (3) the external pipe surface and the saw were disinfected with chlorine; (4) the cut-out pipe was closed as quickly as possible using predisinfected caps, and both the flow direction and the up-down sides of the pipe were marked on the pipe section (Figure S1-B).

In total, 3 pieces of the flushed pipe (length $=30 \mathrm{~cm}$ ) were cut out from each end to sample the biofilm (each piece in duplicate). In total, 6 pieces $(2 \times 3)$ of pipe specimens were taken; 2 pieces were reserved for morphological study by scanning microscopy; and 4 pieces were reserved for microbiological analyses. If the four replicate pipe samples from both ends show the same results, then it is fair to assume that the biofilm is homogeneous along the flow direction for the three swabbed pipe sections in between. The pipe specimen was sealed with predisinfected caps and filled with $1 \mathrm{~L}$ of DNA-free water (Millipore H20MB1006) to preserve the moisture on the internal surface during transport. In this study, DNA-free water was chosen over buffer solutions to accommodate for other potential physiochemical analysis.

2.2.2. Swabbing. For the swab sampling, the $360^{\circ}$ inner ring of the water pipe surface area was divided into 16 parts, grouped into 3 main sections (Figure 1B): Section 1 (S-I) refers to the top half circle of the water pipe surface area (top $180^{\circ}$, accounting for $8 / 16$ parts); Section 2 (S-II) refers to the middle-down part of the water pipe surface area (middle-down $90^{\circ}$, accounting for $4 / 16$ parts); and Section 3 (S-III) refers to the bottom-down part of the water pipe surface area (bottomdown $90^{\circ}$, accounting for $4 / 16$ parts), where most of the loose deposits accumulated. The uneven grouping into the abovedefined 3 sections is based on our experience, built up since 2012, with Dutch distribution systems, in which we observed higher amounts and bigger variations of biofilm in the lower semicircle of pipes (Figure S1-C). This explains why the lower semicircle of the pipe is further divided into two sections (S-II and S-III). Along the flow direction, triplicate cross-sections were sampled; in each of which $8 / 16$ parts were swabbed. To collect representative swab samples from each cross-section, random sampling was applied using an $\mathrm{R}$ script (Table $\mathrm{S} 1$, in total $3 \times 8=24$ swab samples were collected).

All of the samples were transported on ice, and pretreatments were performed in the lab within $2 \mathrm{~h}$ of the sampling at the distribution site. To detach bacteria from swabs and pipe specimens, all the samples were pretreated by low-energy ultrasonic treatment for 3 periods of 2 min each (Branson ultrasonic water bath, $43 \mathrm{kHz}, 180 \mathrm{~W}$ power output, $10 \mathrm{~L}$ sonication chamber). ${ }^{28}$ The obtained suspensions were used for further microbiological analysis. All samples were analyzed within $24 \mathrm{~h}$ after they were taken.

2.3. Scanning Electronic Microscopy. The morphology of biofilm distributed over $360^{\circ}$ of the pipe surface was studied by scanning electron microscopy (SEM) to obtain high resolution images (JEOL JSM-840A). The fixed samples (as described above) were first serially dehydrated with ethanol at concentrations up to $100 \%$, then dried at the critical point, and then sputter-coated with a $10 \mathrm{~nm}$ layer of gold. As previously described, the working distance was $7-39 \mathrm{~mm}$ to achieve higher solution imaging. ${ }^{29}$

2.4. Adenosine Triphosphate (ATP). The ATP concentrations, as a measure for active biomass, were determined for all the samples. The total ATP concentration was determined, as described previously, ${ }^{28,30}$ using the BacTiter Glo reagent and a luminometer. In summary, the obtained suspension was warmed to $30{ }^{\circ} \mathrm{C}$ in a sterile Eppendorf tube, while the ATP reagent was simultaneously warmed. The sample and the reagent were combined after $2 \mathrm{~min}$ at $30{ }^{\circ} \mathrm{C}$ and then the luminescence was measured directly. The data were collected as relative light units and converted to ATP, by means of a calibration curve made with a known ATP standard (BactTiter kit, Promega, method detection limit $0.5 \mathrm{ng} / \mathrm{L}$ ). The recovery of ATP, by both swabbing and sonicating pipe specimens, has been evaluated previously in other studies. ${ }^{19,30,31}$

2.5. DNA Extraction and Illumina 16S rRNA Sequencing. The DNA was extracted from the water samples, and other suspensions obtained after pretreatment, using the FastDNA Spin Kit for Soil (Q-Biogene/MP Biomedicals, Solon, OH, U.S.A.) according to the manufacturer's instructions. 16S rRNA gene amplification was carried out as described. ${ }^{32}$ Briefly, the extracted gDNA was amplified with a primer set (515F: 5'-GTGCCAGCMGCCGCGGTAA-3' and 909R: 5'-CCCGTCAATTCMTTTRAGT-3') targeting the V4-V5 hypervariable regions of sequences from both bacterial and archaeal domains. The primer set was modified for the Illumina Miseq platform (Illumina, Inc., San Diego, CA, U.S.A.), by appending the Illumina sequencing adaptors on the $5^{\prime}$ end. Paired-end sequencing of the amplicons $(2 \times 300 \mathrm{bp})$ was done by BaseClear (Leiden, The Netherlands). The sequencing data have been deposited in the NCBI database, with reference code PRJNA517242; the sample origin of each sequencing library is provided in Table S2.

2.6. Sequences Data Processing. The sequences generated from the Illumina Miseq analysis of the 16S rRNA gene amplicons were processed (i.e., filtered, clustered, and taxonomically assigned and aligned) using the Quantitative Insights Into Microbial Ecology (QIIME2, v2018.6) pipeline with the default settings. ${ }^{33,34}$ Raw sequences were first processed using DADA2, ${ }^{35}$ including quality filtering, denoising, paired-end sequence merging, and chimera filtering. DADA2 generated unique amplicon sequence variants that were equivalent to $100 \%$ similarity operational taxonomic units (OTUs) in the conventional practice. In this publication, we still use the term OTU for the purpose of simplicity. Taxonomy was assigned using q2-feature-classifier, ${ }^{36}$ customized for the primer set used in this study with Silva SSU database release $132 . .^{37}$ Multiple sequence alignment and phylogenetic tree construction were performed using the QIIME 2 plugin q2-phylogeny. Alpha and beta diversity analyses were performed using the QIIME 2 plugin q2diversity. Weighted and unweighted UniFrac distance matrices were constructed from the phylogenetic tree (built by a 

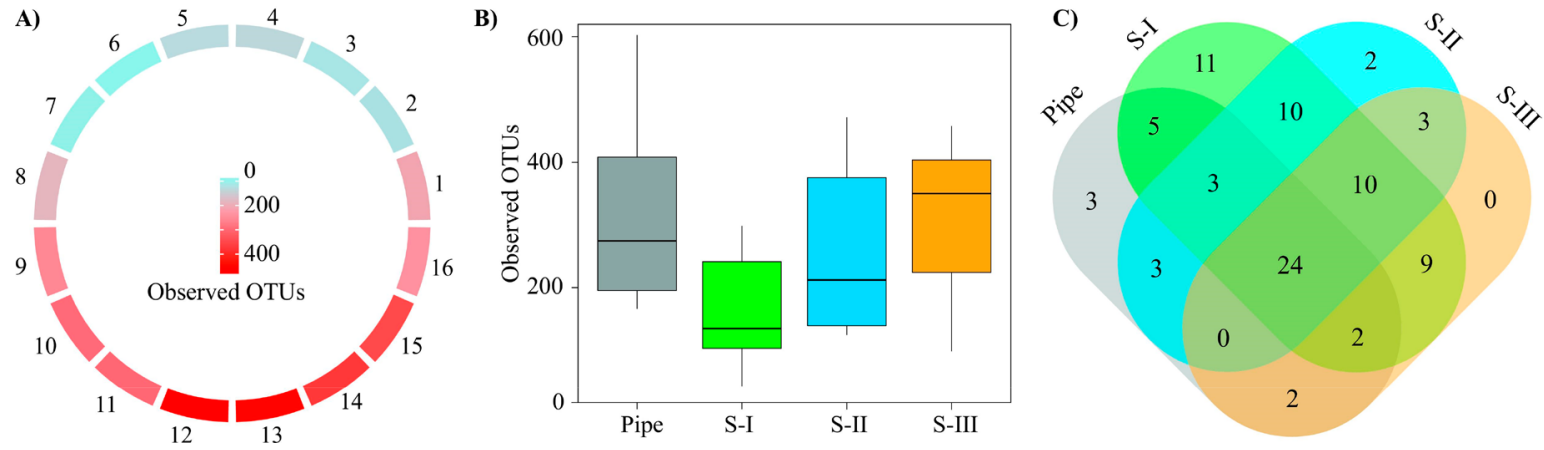

Figure 2. Observed OTUs shown as heatmap (A) and box plot (B): pipe in gray, swab from S-I in green, S-II in blue, and S-III in brown. In the box plot, the whiskers point to the maximum and minimum results; each box shows the upper quartile and lower quartile of the results; the line in the boxes indicates the median of the results. Outliers are shown by dots outside the boxes and whisker range (if existent). (C) Venn plot shows the sharing of dominate OTUs $(>1 \%)$ among the different sample categories.
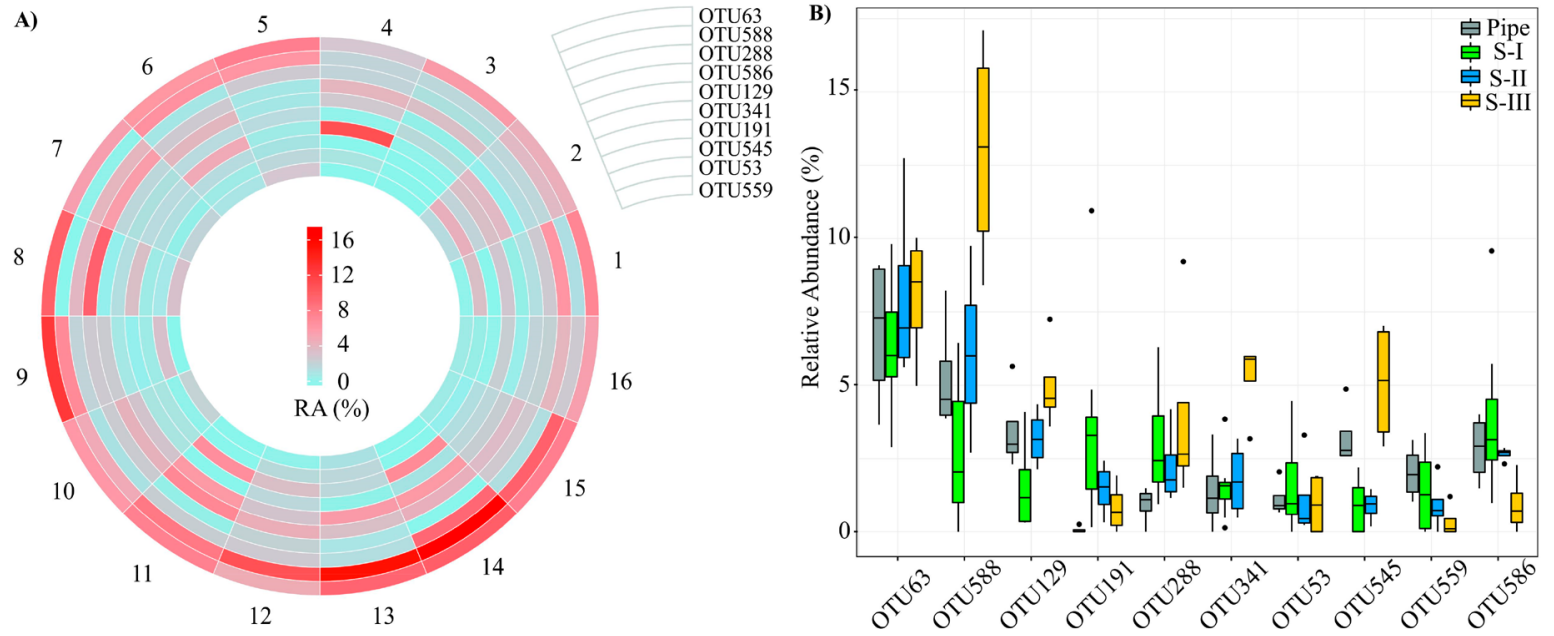

Figure 3. (A) circular heatmap shows the OTU distribution (top 10) over 16 parts; (B) Pipe (B, gray) and swabs taken from S-I in green, S-II in blue, and S-III in brown. For the top 10 OTUs, the assigned taxonomy information is as below: OTU63: P_Acidobacteria; OTU588: Gammaproteobacteria; OTU288: Nitrospira spp.; OTU586: Gammaproteobacteria; OTU129: Terrimonas spp.; OTU341: Gemmataceae; OTU191: Anaerolineae; OTU545: Nitrosomonadaceae; OTU53: Saccharibacillus spp.; OUT 559: Betaproteobacteriales.

FastTree algorithm) and used to conduct a principal coordinate analysis (PCoA). ${ }^{38}$ The core OTUs are defined as the OTUs with a defined cutoff of relative abundance $(>1 \%)$ within each sample and occupancy within each sampling group (fractions of samples that a given OTU was detected, $>80 \%$ ). The shared and unique core OTUs in the biofilms collected by swabbing and pipe section sonication were shown in the Venn diagram (Figure 2C).

2.7. Statistical Analysis. One-way analysis of variance (ANOVA) was used to determine the significance of differences between samples and normally distributed data (affirmed by $Q-Q$ plots, $\chi^{2}$-squared tests, and KolmogorovSmirnov tests). The PERMANOVA test was performed by QIIME2 to determine the significance of beta diversity differences among the sample groups. In both analyses, differences were considered significant when the $p$-value was lower than $0.05(p<0.05)$.

\section{RESULTS}

3.1. Visualization and Quantification of Biofilm Distribution. Regarding the quantitative distribution of biofilm, 16-212 pg ATP $\mathrm{cm}^{-2}$ was detected over the 16 parts of $360^{\circ}$ pipe surfaces. However, as shown by heatmap in Figure $1 \mathrm{C}$, biofilm was not evenly distributed among the 16 parts and 3 sections. Instead, ATP increased as the measurements proceeded down from the top to the bottom of the pipe, suggesting more active biofilm was present at the bottom compared to the top and middle of the pipe. When the ATP data are grouped into the 3 defined sections, the results show that the ATP of S-III was the highest, followed by S-II and S-I (Figure 1D). The differences among the sections were statistically significant $(p<0.01)$. Compared to the biofilm collected by pipe specimen sonication $(91.7 \pm 9.8$ pg ATP $\mathrm{cm}^{-2}$ ), the swab samples yielded comparable ATP levels, but with larger variation $\left(76.5 \pm 57.4 \mathrm{pg}\right.$ ATP $\left.\mathrm{cm}^{-2}\right)$. The SEM pictures of the different parts and sections visually confirmed the differences in biofilm content over the wall of the pipe section, with more biofilm present in the bottom section, while little biofilm was found on the top area of surfaces (Figure 1E$\mathrm{G})$.

3.2. Observed OTUs in Biofilm. The bacterial communities were profiled for all 28 samples (4 pipe specimensonicated biofilm samples and 24 swab biofilm samples). In total, about 560000 sequences were generated, which were assigned to 627 OTUs (taxonomy information on the core OTUs is shown in Table S4). The rarefaction curves reached a plateau after 5000 sequence reads were obtained, indicating the sufficient sample coverage in this study (Figure S2). 
3.2.1. Number of Observed OTUs. The number of observed OTUs is presented for alpha diversity purposes. Over the 16 parts of $360^{\circ}$ pipe surfaces, 40-600 OTUs were observed (Figure 2). As in the case of the ATP results, the observed OTUs were also not distributed evenly; more OTUs were observed as the observations proceeded down from the top to the bottom of the pipe (Figure 2A, circular heatmap). When we grouped the observed OTUs in the 3 defined sections, we observed that S-III contained the highest number of OTUs, followed by S-II, while S-I contained the lowest number (Figure 2B). The differences between the sections are statistically significant $(p<0.01)$. Compared to the biofilm collected by sonication ( $330 \pm 110$ OTUs), the average number of OTUs observed in swab samples was lower $(218 \pm$ 127 OTUs).

Interestingly, more dominant OTUs (relative abundance $>1 \%$, in total 87 OTUs) were present in the swab samples than in the biofilm samples collected by sonication (79 vs 42 OTUs). A closer look at the 3 sections showed that there were 74, 55, and 50 dominant OTUs in S-I, -II, and -III, respectively, which is contrary to the order of total observed OTUs. Among the 87 OTUs, the Venn graph showed that there were 34 OTUs shared by swab biofilm from the 3 sections, 34 OTUs shared by swab biofilm samples and pipe specimen sonicated biofilm samples, of which 24 OTUs were shared by all samples (Figure 2C). There were 16, 2, and 13 unique dominant OTUs within S-I, -II, and -III, respectively. The details of the OTUs' ID in the Venn graph is given in Table S3.

3.2.2. Distribution of Dominant OTUs. The distribution of the top 10 core OTUs (relative abundance $>1 \%$, occupancy $>80 \%$ ) and their taxonomic information are shown in Figure 3. In general, the heatmap showed clearly that all 10 OTUs were distributed unevenly over the 16 parts (Figure 3A), although OTU63 is the only one to be dominant in all 16 parts $(7.2 \pm$ $2.4 \%)$. Grouping the distribution of dominant OTUs and their relative abundance according to the 3 defined sections, the average relative abundance of OTU588 and OTU129 increased clearly when observations proceeded down from the top to the bottom of the pipe $(\mathrm{S}-\mathrm{I}<\mathrm{S}$-II $<$ S-III), while the relative abundance of OTU191 and OTU559 decreased (S-I > S-II > S-III) (Figure 3B). A clear trend was observed for a number of OTUs, that is, they concentrated in the direction of the pipe bottom (S-III). For example, 5/10 dominant OTUs showed significantly higher relative abundance in S-III compared to the other two sections (e.g., OTU63, OTU588, OTU129, OTU341, and OTU545). Compared to the swab samples, the relative abundances of the top 10 dominant OTUs within biofilm samples collected by pipe specimen sonication were mostly at a level around the mean value of S-I, S-II, and S-III.

3.3. Bacterial Community Composition. The taxonomy information on the above-mentioned core OTUs is given in Table S4. At the phylum level, all the biofilm samples were dominated by Proteobacteria (56.5 $\pm 9 \%$ ), followed by Acidobacteria $(17.7 \pm 6.9 \%)$, Bacteroidetes $(7.9 \pm 5.6 \%)$, Chloroflexi $(4.6 \pm 3.2 \%)$, Gemmatimonadetes $(2.8 \pm 2.1 \%)$, Actinobacteria $(2.7 \pm 2.1 \%)$, and Nitrospira $(2.8 \pm 2.5 \%)$ (Figure 4). For the swab biofilm samples over 16 parts of the $360^{\circ}$ pipe surfaces, Proteobacteria accounted for $40.8-71.1 \%$. No clear differences were observed for Proteobacteria, neither among samples from different sections nor between biofilm samples collected by swabbing and pipe specimen sonication.

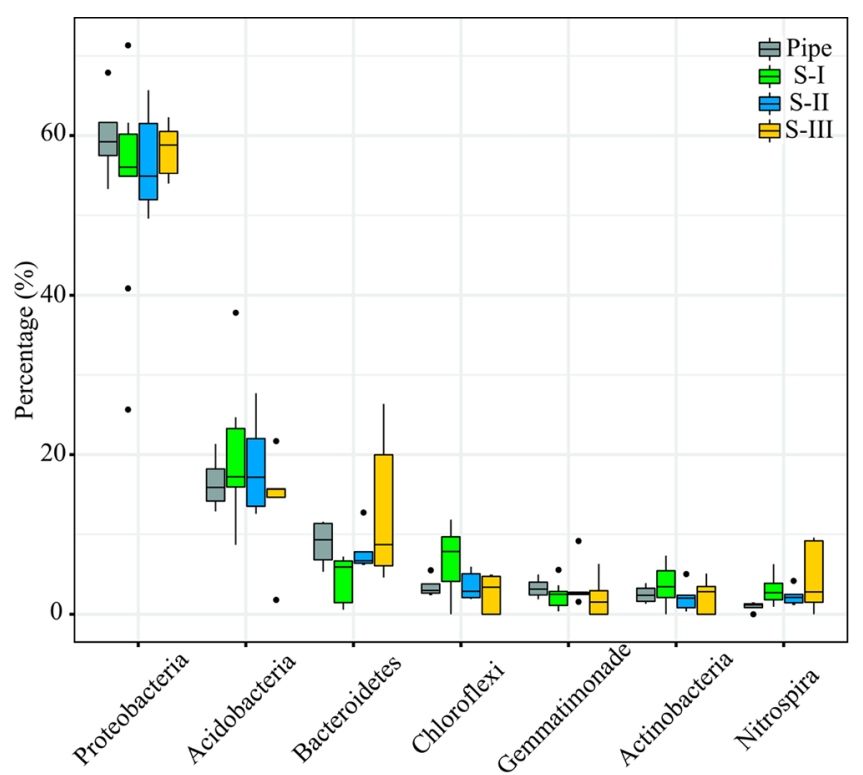

Figure 4. Boxplot showing the dominate phylum $(>1 \%)$ among the different sample categories (Pipe: gray; swabs from S-I in green, S-II in blue, and S-III in brown).

It was observed that the relative abundance of Chloroflexi was higher in S-I compared to S-II and S-III, whereas Bacteroidetes and Nitrospira were relatively more highly abundant in S-III than in S-II and S-I.

Not all OTUs were assigned to genus level; for example, OTU63 (which dominated all samples) was only assigned to the phylum level: Acidobacteria. Among the dominant genera, Nitrospira spp. (2.9-4.6\%), Terrimonas spp. (1.8-3.5\%) and Hyphomicrobium spp. (1.2-3.3\%) were dominant in all sections, while Saccharibacillus spp. $(1.1-1.7 \%)$ were shared by S-I and S-III (Figure S2). Gaiella spp. (1.2\%) and Vicinamibacter spp. (1.1\%) and presented mainly in S-I, Blastopirellula spp. (2.5\%), and Pirellula spp. (1.2\%) dominated in S-II, while Holophaga spp. (6.1\%) and Phaeodactylibacter spp. (4.1\%) was relatively abundant in S-III.

3.4. Beta Diversity Comparing Bacterial Community Similarity. The beta diversity results are represented in a PCoA plot (Figure 5). The high level of similarity of replicate samples attests to the quality of the sampling conducted in this study, and the reproducibility of subsequent $16 \mathrm{~S}$ rRNA gene sequencing and the obtained results.

As shown in Figure 5, most of the samples clustered together, with several samples scattered around. A comparison of the biofilm samples collected by pipe specimen sonication and the swabs, shows the pipe specimens clustered much closer to each other, indicating the better reproducibility of pipe specimen samples and the larger variations in the swab samples in the same section and between sections. For the swab samples, the biofilm in S-II clustered closely together, while the cluster of the S-I samples was much looser, and the S-III samples showed the largest variations. The PERMANOVA test by QIIME2 showed significant differences between sample groups of pipe specimen sonication and swabbing $(p<0.001)$, and significant variations among swabs from the different sections $(p<0.05)$. 


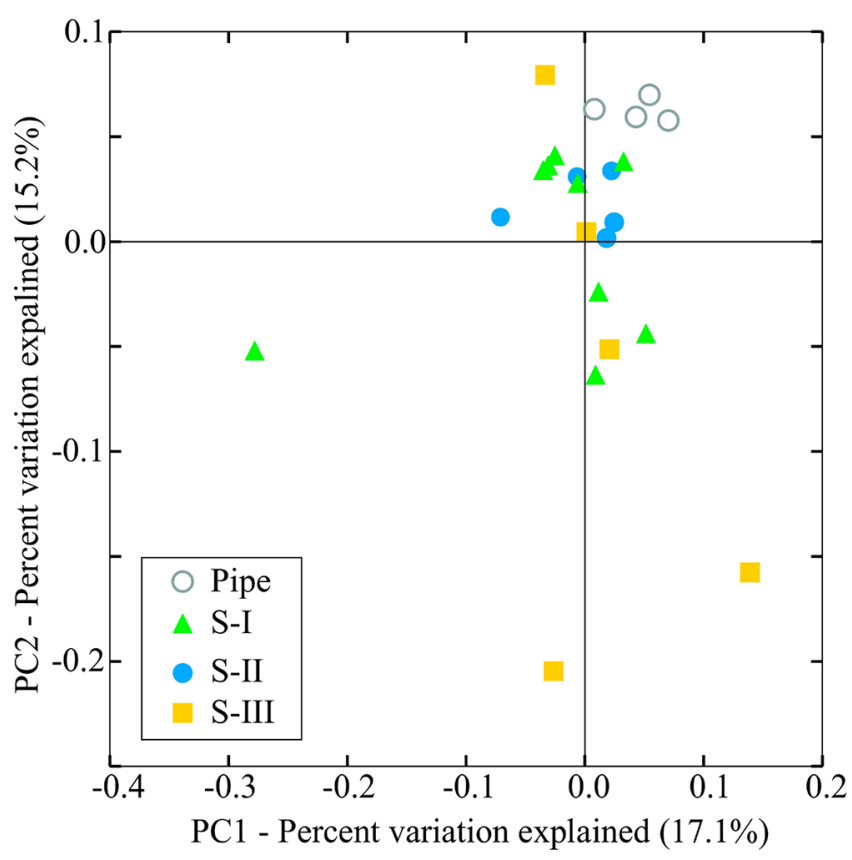

Figure 5. PCoA plot illustrates the similarity of bacterial community collected by cutting pipe sections for sonication (pipe, gray) and swabs from different sections over $360^{\circ}$ (S-I: green; S-II: blue; S-III brown).

\section{DISCUSSION}

Other studies have been conducted and valuable knowledge has been reported regarding the spatial distribution of biofilm across drinking water systems, for example, biofilters, ${ }^{39}$ and field and simulated distribution systems..$^{27,40,41}$ To the best of our knowledge, this is the first study to investigate the $360^{\circ}$ radial variation of biofilm in a cross-section of operational drinking water distribution pipes, and to compare the sampling approaches of swabbing and sonicating pipe specimens. Our results reveal an uneven distribution of both biofilm quantity and community over the $360^{\circ}$. This is critical for a scientific understanding of the microbiological processes during drinking water distribution. It is also essential for the development of practical management strategies and policies to ensure biosafe and high-quality drinking water for consumers, in view of the consistent release of cells from biofilm into bulk water. ${ }^{5}$ Moreover, the new knowledge can be used by utilities and public agencies to guide their sampling of drinking water biofilms for research and monitoring purposes, especially when they use swabbing to sample the biofilm.

4.1. Uneven Radial Distribution of Drinking Water Biofilm. The SEM images make clear that more material accumulates as the scan proceeds down from the top to the bottom of the pipe. Quantitatively, 16-212 pg ATP $\mathrm{cm}^{-2}$ of biofilm was detected in all the samples. The obtained results are within the typical ranges of (Dutch) drinking water biofilms. ${ }^{2,42}$ These values are in the same range of our previous study of other Dutch drinking water systems (90-160 pg ATP $\mathrm{cm}^{-2}$ ), in which the biofilm samples were also collected by pipe specimen sonication. ${ }^{19,22}$ The larger range of ATP results observed in this study reflected the different position on the pipe where the biofilm was taken by swabbing, because the results obtained by pipe specimen sonication were relatively stable $\left(91.7 \pm 9.8 \mathrm{pg}\right.$ ATP $\left.\mathrm{cm}^{-2}\right)$. Among the predefined 16 parts and 3 sections, a clear increase in ATP concentration was observed as one proceeds down from the top to the bottom of the pipe; the differences are statistically significant $(P<0.01)$, suggesting more active biomass concentration at the bottom half of the pipe, especially in S-III of the bottom. This may be because of the accumulation of particles and particle-associated bacteria in the pipe bottom in the biofilm matrix with the help of biofilm EPS. ${ }^{43}$ For S-III of the pipe bottom, it appears that despite the hydraulic turbulence and reoccurring particle resuspension, gravity drives more particle-associated bacteria (PAB) accumulation (S-III, part 11, 12, 13, and 14), ${ }^{44-46}$ which gradually aggregates and builds up, and finally cannot be resuspended by regular hydraulic peaks (e.g., morning or evening peaks). ${ }^{19}$ The types and properties of particles (e.g., size, weight) in the distribution system are critical with regards to any impact gravity might have. For example, for particles with an average size of $0.010 \mathrm{~mm}$ (significant portion $<0.005$ $\mathrm{mm}$ ), the lowest flow velocities in the distribution system would suffice to overcome the gravity settling forces. ${ }^{47}$ However, some larger and heavier particles do accumulate and are retained in the distribution system, albeit in very low proportions. These loose deposits cannot be transferred back into the bulk water during regular operations, but during the flushing of the network for hydraulic cleaning. Regardless of the type concerned, once the particles reach the bottom, the formed biofilm matrix will retain them and incorporate them into the biofilm. As a result, even after the loose deposits are flushed out, the biofilm present at the pipe bottom is higher than in other pipe sections.

Although loose deposits were not covered in the present study, they have been found to constitute reservoirs for $\mathrm{Fe}$, $\mathrm{Mn}$, organic matter, and bacteria, ${ }^{22,46,48}$ so their removal can significantly improve distribution system water quality. ${ }^{49}$ For their part, the attached bacteria and available nutrients from the accumulated particulate matter can promote biofilm growth. $^{22,24}$

As in the case of ATP, the number of observed OTUs increased as the observations proceeded down from the top to the bottom of the pipe, indicating a higher diversity of biofilm community at S-III than at S-II and S-I (ranked S-III > S-II > $\mathrm{S}-\mathrm{I}$ ). Again, this higher diversity at the pipe bottom is ascribed to the contribution from loose deposits, because loose deposits mainly concentrate at the pipe bottom and have been reported to harbor a different bacterial community than the biofilm. ${ }^{19,50}$ In contrast, when focusing on the dominant OTUs (>1\%), we found that there were fewer OTUs, with higher relative abundances in S-III than in the other sections (ranked S-III < S-II $<$ S-I). This may be the result of higher concentrations and different composition of nutrients, as well as the multiple aerobic and anoxic microenvironments provided by loose deposits, which stimulate certain groups of bacteria to take a dominant role while inhibiting others. ${ }^{19}$ This theory is supported by the taxonomy profile of the shared dominant genera (Nitrospira spp., Terrimonas spp., and Hyphomicrobium spp.), which have been widely found in drinking water environments, ${ }^{19,51,52}$ while the dominant genus observed in SIII, Holophaga spp. (6.1\%), contains a single species of Holophaga fetida, which is obligately anaerobic. ${ }^{53}$ Whereas, the results of Shannon index and Pielou-e revealed that there were no significant differences in bacterial community evenness among sampling groups (Figure S3).

4.2. Comparison between Swabbing and Pipe Specimen Sonication. In contrast to the swab samples, the 4 samples collected by sonicating pipe specimens showed minor 
variations regarding the ATP values and the composition and diversity of the bacterial communities. This suggested a homogeneous distribution of biofilm along the flow direction. This observation accorded with previous field drinking water biofilm studies in The Netherlands and Germany. ${ }^{16,19}$ Our study of another unchlorinated Dutch drinking water supply system found the same homogeneous biofilm core communities across different locations. ${ }^{19}$ While in the chlorinated German drinking water system, large spatial variations were observed among swab biofilm samples from different locations (the swabbing position on the pipe surface was not described); but at the same location, Henne et al. found that the biofilm core communities corresponded even more with the vicinity of biofilm than with the types of support material. ${ }^{16}$ In the present study, the lower variation among pipe specimen sonicated samples compared to the swab samples can be explained by the coverage of all the 3 sections, and the much bigger surface area $\left(942 \mathrm{~cm}^{2}\right.$ vs $\left.4 \mathrm{~cm}^{2}\right)$. In other words, the sampling of $30 \mathrm{~cm}$ pipe specimens can overcome the potential influences induced by selecting the positions for swabbing 4 $\mathrm{cm}^{2}$ pipe surface.

4.3. Sampling Strategy for Collecting Drinking Water Biofilm. There is no doubt that it is critical to take representative samples of good quality for any kind of drinking water biofilm study, otherwise the obtained results may lead to a misunderstanding of the biofilm process. In light of the growth of (opportunistic) pathogens in the biofilm and the continuous biomass exchange between biofilm and bulk water, a failure to determine the real situation of biofilm poses a potential biorisk to the microbiological water quality and ultimately to consumer health.

When one examines the literature, it is clear that swabbing has been used as the main sampling methodology for collecting biofilm samples over the last decades. ${ }^{30,54-57}$ Our experience with cutting pipe specimens for sonication to collect biofilm samples from operational PVC-U pipes distributing drinking water is relatively recent. ${ }^{8,19,22}$ On the basis of the above discussion, especially considering the significant quantitative and qualitative variations among swab samples from 3 sections, it seems that cutting pipe specimens for sonication is a better biofilm sampling method for achieving reproduceable results. However, the limitations of pipe specimen sonication are also clear: (1) the opportunity to assess field distribution system is very limited; ${ }^{11}$ (2) when the field distribution system is accessible, the cutting out of pipe sections requires the suspension of the water supply to connected customers, and the subsequent close monitoring of the hygienic conditions ${ }^{46}$ and the possibility of increased metal release (e.g., $\mathrm{Pb}$ ) from the pipe material; ${ }^{58}$ (3) after collecting the pipe specimens, large pipes cannot be pretreated by sonication as a whole piece, because they are too big for the sonication water bath. In such cases, the common practice is to sample the biofilm by swabbing/scratching the pipe surface. ${ }^{59}$ Another important point is that the sectional variations, the process of biofilm development, and the real distribution system's microbial ecology will be obscured because of the big surface area and multisections being covered.

Therefore, swabbing will still be the inescapable option for taking drinking water biofilm from field distribution systems in most cases. However, this first study investigating the $360^{\circ}$ distribution of drinking water biofilm in an operational system clearly illustrates the uneven distribution of biofilm, as a result of which the swab samples from different positions and sections will be difficult to compare, and the cross-comparison of samples may lead to misunderstandings of distribution system microbial ecology. This is especially true when the targets are large-diameter transportation pipes, with water containing higher loads of particulate matter because of the pipe water velocity profile and the radial behavior of particles in water pipes. ${ }^{60-62}$ Yet, until now, only a few drinking-water biofilm process studies have given any positions around the circumference of the pipe surface where the swabs were taken. ${ }^{18}$ The present paper is the first, to the best of our knowledge, to provide details on the entire circumference.

Clearly, it is critical that a swabbing strategy be developed that can overcome the variations induced by swabbing positions, and avoid potential misunderstandings of the results of cross-sectional comparisons. On the basis of the findings from the present study, the following consistent multisectional swabbing strategy is proposed: (1) the swabbing positions should be recorded and described in the study; (2) swabs from all three sections should be collected; (3) consistent swabbing should be done from the same positions at different locations; and (4) while processing the results, comparisons should be made between swabs from the same position/section from different locations, and different positions/sections from the same location. Such a swabbing strategy can reveal the radial and spatial distribution of biofilm, uncover the origin and development of microbial ecology, and offer solid evidence to develop efficient biological water quality management strategies in drinking water distribution pipes.

In summary, clear variations were observed for the biofilm over the $360^{\circ}$ of the surface of real water distribution networks. The comparison of pipe specimen sonication and swab samples offers valuable insight into the sampling strategies to collect representative biofilm samples from field distribution systems. According to the present study, the biofilm is unevenly distributed over the 16 parts of the $360^{\circ}$; the active biomass, as measured by ATP, increased as measurements proceeded down from the top to the bottom of the pipe. Similarly, the number of observed OTUs increased as observations proceeded down from the top to the bottom of the pipe. In contrast, the number of dominant OTUs $(>1 \%)$ was lowest at the pipe bottom. Regarding the bacterial communities, all sections were dominated by Proteobacteria. At the genus level, Nitrospira spp., Terrimonas spp., and Hyphomicrobium spp. were dominant in all sections. Gaiella spp. and Vicinamibacter spp. dominated in S-I, Blastopirellula spp. and Pirellula spp. dominated in S-II, while Holophaga spp. and Phaeodactylibacter spp. dominated in S-III. The comparison of swabbing and pipe specimen sonication shows that the sampling strategy has a significant influence on the biofilm bacterial community obtained (based on the observed OTUs, $P<0.001)$. For future biofilm sampling, we propose that a consistent, multisectional swabbing strategy be applied to collect samples from all sections, and that the swabs be compared from the same position/section across locations. Our results will be especially valuable for water utilities and public agencies in guiding their biofilm research and monitoring. The inadequate and/or incorrect biofilm sampling can result in misunderstandings about biofilm formation and microbial ecology in drinking water distribution systems, masking the potential microbiological water quality risks resulting from cell transfer between biofilm and bulk water, ${ }^{5,83}$ and holding back the development of regulations, 
policies, or management strategies to safeguard public health associated with drinking water.

\section{ASSOCIATED CONTENT}

\section{SI Supporting Information}

The Supporting Information is available free of charge at https://pubs.acs.org/doi/10.1021/acs.est.9b06603.

Table S1, Random sampling calculated by R script for the three swabbed cross-sections; Table S2, meta information of sequencing data deposited in NCBI database; Table S3, detailed information of OTUs' ID presented in the Venn graph; Table S4, assigned taxonomy profile of the core OTUs; Table S5, dominant genera in all sample types; Figure $S 1$, on-site pipe cutting in the field and examples of the inner pipe surface condition after cutting open the pipe; Figure S2, rarefaction curve showing the number of observed OTUs and the sequence depth; and Figure S3, Shannon index and Pielou-e results showed by box plot (PDF)

\section{AUTHOR INFORMATION}

\section{Corresponding Author}

Gang Liu - Key Laboratory of Drinking Water Science and Technology, Research Centre for Eco-Environmental Sciences, Chinese Academy of Sciences, Beijing 100085, P. R. China; Oasen Drinkwater, 2801SB Gouda, The Netherlands; Sanitary Engineering, Department of Water Management, Faculty of Civil Engineering and Geosciences, Delft University of Technology, 2600GA Delft, The Netherlands; ○ orcid.org/ 0000-0002-4008-9017; Phone: 0086 17600879707; Email: gliu@rcees.ac.cn, g.liu-1@tudelft.nl

\section{Authors}

Ya Zhang - Department of Civil and Environmental Engineering, University of Illinois at Urbana-Champaign, Urbana, Illinois 61801, United States

Xinlei Liu - Catalysis Engineering, Chemical Engineering Department, Delft University of Technology, $2629 \mathrm{HZ}$ Delft, The Netherlands; 이이이.org/0000-0001-7552-1597

Frederik Hammes - Eawag, Swiss Federal Institute of Aquatic Science and Technology, Dübendorf, Switzerland

Wen-Tso Liu - Department of Civil and Environmental Engineering, University of Illinois at Urbana-Champaign, Urbana, Illinois 61801, United States

Gertjan Medema - Sanitary Engineering, Department of Water Management, Faculty of Civil Engineering and Geosciences, Delft University of Technology, 2600GA Delft, The Netherlands; KWR Watercycle Research Institute, 3430 BB Nieuwegein, The Netherlands

Peter Wessels - Oasen Drinkwater, 2801SB Gouda, The Netherlands

Walter van der Meer - Oasen Drinkwater, 2801SB Gouda, The Netherlands; Science and Technology, University of Twente, 7500AE Enschede, The Netherlands; O orcid.org/0000-00029278-840X

Complete contact information is available at: https://pubs.acs.org/10.1021/acs.est.9b06603

\section{Notes}

The authors declare no competing financial interest.

\section{ACKNOWLEDGMENTS}

The authors would like to acknowledge the support from the National Key R\&D program of China (2018YFE0204100), National Natural Science Foundation of China for International Cooperation and Exchange (51820105011), and the "Hundred Talents Program" of the Chinese Academy of Sciences.

\section{REFERENCES}

(1) Proctor, C. R.; Hammes, F. Drinking water microbiology-from measurement to management. Curr. Opin. Biotechnol. 2015, 33, 8794.

(2) Liu, G.; Verberk, J. Q. J. C.; Van Dijk, J. C. Bacteriology of drinking water distribution systems: an integral and multidimensional review. Appl. Microbiol. Biotechnol. 2013, 97 (21), 9265-9276.

(3) Wang, H.; Masters, S.; Edwards, M. A.; Falkinham, J. O.; Pruden, A. Effect of Disinfectant, Water Age, and Pipe Materials on Bacterial and Eukaryotic Community Structure in Drinking Water Biofilm. Environ. Sci. Technol. 2014, 48 (3), 1426-1435.

(4) Chaves Simões, L.; Simões, M. Biofilms in drinking water: Problems and solutions. RSC Adv. 2013, 3 (8), 2520-2533.

(5) Chan, S.; Pullerits, K.; Keucken, A.; Persson, K. M.; Paul, C. J.; Rådström, P. Bacterial release from pipe biofilm in a full-scale drinking water distribution system. NPJ. biofilms and microbiomes 2019, 5 (1), 9.

(6) LeChevallier, M. W.; Babcock, T. M.; Lee, R. G. Examination and characterization of distribution system biofilms. Appl. Environ. Microbiol. 1987, 53 (12), 2714-2724.

(7) Silhan, J.; Corfitzen, C. B.; Albrechtsen, H. J. Effect of Temperature and Pipe Material on Biofilm Formation and Survival of Escherichia coli in Used Drinking Water Pipes: A Laboratory-Based Study; In Rose, J.; Medema, G., Eds.; 2006; Vol. 54, pp 49-56.

(8) Liu, G.; Zhang, Y.; van der Mark, E.; Magic-Knezev, A.; Pinto, A.; van den Bogert, B.; Liu, W.; van der Meer, W.; Medema, G. Assessing the origin of bacteria in tap water and distribution system in an unchlorinated drinking water system by SourceTracker using microbial community fingerprints. Water Res. 2018, 138, 86-96.

(9) Momba, M. N. B.; Kfir, R.; Venter, S. N.; Cloete, T. E. An overview of biofilm formation in distribution systems and its impact on the deterioration of water quality. Water SA 2000, 26 (1), 59-66.

(10) Liu, G.; Zhang, Y.; Knibbe, W.-J.; Feng, C.; Liu, W.; Medema, G.; van der Meer, W. Potential impacts of changing supply-water quality on drinking water distribution: A review. Water Res. 2017, 116, 135-148.

(11) Berry, D.; Xi, C.; Raskin, L. Microbial ecology of drinking water distribution systems. Curr. Opin. Biotechnol. 2006, 17 (3), 297-302.

(12) Van der Kooij, D. Potential for biofilm development in drinking water distribution systems. Journal of Applied Microbiology Symposium Supplement 1999, 85 (28), 39S-44S.

(13) Eisnor, J. D.; Gagnon, G. A. A framework for the implementation and design of pilot-scale distribution systems. Aqua 2003, 52 (7), 501-519.

(14) Hong, P. Y.; Hwang, C.; Ling, F.; Andersen, G. L.; LeChevallier, M. W.; Liu, W. T. Pyrosequencing analysis of bacterial biofilm communities in water meters of a drinking water distribution system. Appl. Environ. Microbiol. 2010, 76 (16), 5631-5635.

(15) Schmeisser, C.; Stöckigt, C.; Raasch, C.; Wingender, J.; Timmis, K. N.; Wenderoth, D. F.; Flemming, H. C.; Liesegang, H.; Schmitz, R. A.; Jaeger, K. E.; Streit, W. R. Metagenome Survey of Biofilms in Drinking-Water Networks. Appl. Environ. Microbiol. 2003, 69 (12), 7298-7309.

(16) Henne, K.; Kahlisch, L.; Brettar, I.; Höfle, M. G. Analysis of structure and composition of bacterial core communities in mature drinking water biofilms and bulk water of a citywide network in Germany. Appl. Environ. Microbiol. 2012, 78 (10), 3530-3538.

(17) Douterelo, I.; Jackson, M.; Solomon, C.; Boxall, J. Microbial analysis of in situ biofilm formation in drinking water distribution 
systems: implications for monitoring and control of drinking water quality. Appl. Microbiol. Biotechnol. 2016, 100 (7), 3301-3311.

(18) Fish, K. E.; Collins, R.; Green, N. H.; Sharpe, R. L.; Douterelo, I.; Osborn, A. M.; Boxall, J. B. Characterisation of the physical composition and microbial community structure of biofilms within a model full-scale drinking water distribution system. PLoS One 2015, 10 (2), e0115824.

(19) Liu, G.; Bakker, G.; Li, S.; Vreeburg, J.; Verberk, J.; Medema, G.; Liu, W.; Van Dijk, J. Pyrosequencing reveals bacterial communities in unchlorinated drinking water distribution system: an integral study of bulk water, suspended solids, loose deposits, and pipe wall biofilm. Environ. Sci. Technol. 2014, 48 (10), 5467-5476.

(20) Douterelo, I.; Sharpe, R.; Boxall, J. Influence of hydraulic regimes on bacterial community structure and composition in an experimental drinking water distribution system. Water Res. 2013, 47 (2), 503-516.

(21) Yu, J.; Kim, D.; Lee, T. Microbial diversity in biofilms on water distribution pipes of different materials. Water Sci. Technol. 2010, 61 (1), 163-171.

(22) Liu, G.; Tao, Y.; Zhang, Y.; Lut, M.; Knibbe, W.-J.; van der Wielen, P.; Liu, W.; Medema, G.; van der Meer, W. Hotspots for selected metal elements and microbes accumulation and the corresponding water quality deterioration potential in an unchlorinated drinking water distribution system. Water Res. 2017, 124, 435445.

(23) Liu, J.; Chen, H.; Yao, L.; Wei, Z.; Lou, L.; Shan, Y.; Endalkachew, S.-D.; Mallikarjuna, N.; Hu, B.; Zhou, X. The spatial distribution of pollutants in pipe-scale of large-diameter pipelines in a drinking water distribution system. J. Hazard. Mater. 2016, 317, 2735 .

(24) Hijnen, W.; Schurer, R.; Bahlman, J.; Ketelaars, H.; Italiaander, R.; van der Wal, A.; van der Wielen, P. Slowly biodegradable organic compounds impact the biostability of non-chlorinated drinking water produced from surface water. Water Res. 2018, 129, 240-251.

(25) Donlan, R.; Pipes, W.; Yohe, T. Biofilm formation on cast iron substrata in water distribution systems. Water Res. 1994, 28 (6), 1497-1503.

(26) Chang, Y.-W.; Fragkopoulos, A. A.; Marquez, S. M.; Kim, H. D.; Angelini, T. E.; Fernández-Nieves, A. Biofilm formation in geometries with different surface curvature and oxygen availability. New J. Phys. 2015, 17 (3), 033017.

(27) Liu, J.; Ren, H.; Ye, X.; Wang, W.; Liu, Y.; Lou, L.; Cheng, D.; $\mathrm{He}, \mathrm{X}$.; Zhou, X.; Qiu, S.; et al. Bacterial community radial-spatial distribution in biofilms along pipe wall in chlorinated drinking water distribution system of East China. Appl. Microbiol. Biotechnol. 2017, 101 (2), 749-759.

(28) Magic-Knezev, A.; van der Kooij, D. Optimisation and significance of ATP analysis for measuring active biomass in granular activated carbon filters used in water treatment. Water Res. 2004, 38 (18), 3971-3979.

(29) Liu, G.; Ling, F.; Magic-Knezev, A.; Liu, W.; Verberk, J. Q. J. C.; Van Dijk, J. C. Quantification and identification of particle associated bacteria in unchlorined drinking water from three treatment plants by cultivation-independent methods. Water Res. 2013, 47 (10), 3523-3533.

(30) Ismail, R.; Aviat, F.; Michel, V.; Le Bayon, I.; Gay-Perret, P.; Kutnik, M.; Fédérighi, M. Methods for recovering microorganisms from solid surfaces used in the food industry: a review of the literature. Int. J. Environ. Res. Public Health 2013, 10 (11), 61696183.

(31) Moore, G.; Griffith, C. Factors influencing recovery of microorganisms from surfaces by use of traditional hygiene swabbing. Dairy Food and Environmental Sanitation 2002, 22 (6), 410-421.

(32) Kozich, J. J.; Westcott, S. L.; Baxter, N. T.; Highlander, S. K.; Schloss, P. D. Development of a dual-index sequencing strategy and curation pipeline for analyzing amplicon sequence data on the MiSeq Illumina sequencing platform. Appl. Environ. Microbiol. 2013, 79 (17), $5112-5120$.
(33) Caporaso, J. G.; Kuczynski, J.; Stombaugh, J.; Bittinger, K.; Bushman, F. D.; Costello, E. K.; Fierer, N.; Pẽna, A. G.; Goodrich, J. K.; Gordon, J. I.; et al. QIIME allows analysis of high-throughput community sequencing data. Nat. Methods 2010, 7 (5), 335-336.

(34) Bolyen, E.; Rideout, J. R.; Dillon, M. R.; Bokulich, N. A.; Abnet, C.; Al-Ghalith, G. A.; Alexander, H.; Alm, E. J.; Arumugam, M.; Asnicar, F. QIIME 2: Reproducible, Interactive, Scalable, and Extensible Microbiome Data Science; 2167-9843; PeerJ Preprints: 2018.

(35) Callahan, B. J.; McMurdie, P. J.; Rosen, M. J.; Han, A. W.; Johnson, A. J. A.; Holmes, S. P. DADA2: high-resolution sample inference from Illumina amplicon data. Nat. Methods 2016, 13 (7), 581.

(36) Bokulich, N. A.; Kaehler, B. D.; Rideout, J. R.; Dillon, M.; Bolyen, E.; Knight, R.; Huttley, G. A.; Caporaso, J. G. Optimizing taxonomic classification of marker-gene amplicon sequences with QIIME 2's q2-feature-classifier plugin. Microbiome 2018, 6 (1), 90.

(37) Quast, C.; Pruesse, E.; Yilmaz, P.; Gerken, J.; Schweer, T.; Yarza, P.; Peplies, J.; Glöckner, F. O. The SILVA ribosomal RNA gene database project: improved data processing and web-based tools. Nucleic Acids Res. 2012, 41 (D1), D590-D596.

(38) Liu, G.; Bakker, G. L.; Li, S.; Vreeburg, J. H. G.; Verberk, J. Q. J. C.; Medema, G. J.; Liu, W. T.; Van Dijk, J. C. Pyrosequencing Reveals Bacterial Communities in Unchlorinated Drinking Water Distribution System: An Integral Study of Bulk Water, Suspended Solids, Loose Deposits, and Pipe Wall Biofilm. Environ. Sci. Technol. 2014, 48 (10), 5467-5476.

(39) Pinto, A. J.; Xi, C.; Raskin, L. Bacterial community structure in the drinking water microbiome is governed by filtration processes. Environ. Sci. Technol. 2012, 46 (16), 8851-8859.

(40) Pinto, A. J.; Schroeder, J.; Lunn, M.; Sloan, W.; Raskin, L. Spatial-Temporal Survey and Occupancy-Abundance Modeling to Predict Bacterial Community Dynamics in the Drinking Water Microbiome. mBio 2014, 5 (3), e01135-14.

(41) Morin, P.; Camper, A. K. Attachment and fate of carbon fines in simulated drinking water distribution system biofilms. Water Res. 1997, 31 (3), 399-410.

(42) Prest, E. I.; Hammes, F.; van Loosdrecht, M. C.; Vrouwenvelder, J. S. Biological Stability of Drinking Water: Controlling Factors, Methods, and Challenges. Front. Microbiol. 2016, 7, 45 .

(43) Flemming, H.-C.; Wingender, J. The biofilm matrix. Nat. Rev. Microbiol. 2010, 8 (9), 623-633.

(44) Vreeburg, J. H. G.; Boxall, D. J. B. Discolouration in potable water distribution systems: A review. Water Res. 2007, 41 (3), 519529.

(45) Matsui, Y.; Yamagishi, T.; Terada, Y.; Matsushita, T.; Inoue, T. Suspended particles and their characteristics in water mains: Developments of sampling methods. Aqua 2007, 56 (1), 13-24.

(46) Gauthier, V.; Gérard, B.; Portal, J. M.; Block, J. C.; Gatel, D. Organic matter as loose deposits in a drinking water distribution system. Water Res. 1999, 33 (4), 1014-1026.

(47) Boxall, J.; Skipworth, P.; Saul, A. A novel approach to modelling sediment movement in distribution mains based on particle characteristics. Water Software Systems 2001, 1, 263-273.

(48) Smith, S. E.; Bisset, A.; Colbourne, J. S.; Holt, D. M.; Lloyd, B. $\mathrm{J}$. The occurrence and significance of particles and deposits in a drinking water distribution system. J. New Engl. Water Works Assoc. 1997, 111 (2), 135-144.

(49) Lehtola, M. J.; Nissinen, T. K.; Miettinen, I. T.; Martikainen, P. J.; Vartiainen, T. Removal of soft deposits from the distribution system improves the drinking water quality. Water Res. 2004, 38 (3), 601-610.

(50) van der Wielen, P. W.; Lut, M. C. Distribution of microbial activity and specific microorganisms across sediment size fractions and pipe wall biofilm in a drinking water distribution system. Water Sci. Technol.: Water Supply 2016, 16 (4), 896-904.

(51) Liao, X.; Chen, C.; Chang, C. H.; Wang, Z.; Zhang, X.; Xie, S. Heterogeneity of microbial community structures inside the up-flow 
biological activated carbon (BAC) filters for the treatment of drinking water. Biotechnol. Bioprocess Eng. 2012, 17 (4), 881-886.

(52) Douterelo, I.; Husband, S.; Loza, V.; Boxall, J. Dynamics of biofilm re-growth in drinking water distributions systems. Appl. Environ. Microbiol. 2016, 82 (14), 4155-4168.

(53) Kreft, J. U.; Schink, B. O-Demethylation by the homoacetogenic anaerobe Holophaga foetida studied by a new photometric methylation assay using electrochemically produced cob (I) alamin. Eur. J. Biochem. 1994, 226 (3), 945-951.

(54) Leadbetter, E. R.; Poindexter, J. S. Methods and Special Applications in Bacterial Ecology; Springer Science \& Business Media: 1985; Vol. 2.

(55) Kotlarz, N.; Rockey, N.; Olson, T. M.; Haig, S.-J.; Sanford, L.; LiPuma, J. J.; Raskin, L. Biofilms in Full-Scale Drinking Water Ozone Contactors Contribute Viable Bacteria to Ozonated Water. Environ. Sci. Technol. 2018, 52 (5), 2618-2628.

(56) Stüken, A.; Haverkamp, T. H.; Dirven, H. A.; Gilfillan, G. D.; Leithaug, M.; Lund, V. Microbial Community Composition of Tap Water and Biofilms Treated with or without Copper-Silver Ionization. Environ. Sci. Technol. 2018, 52 (6), 3354-3364.

(57) Van Nevel, S.; Buysschaert, B.; De Roy, K.; De Gusseme, B.; Clement, L.; Boon, N. Flow cytometry for immediate follow-up of drinking water networks after maintenance. Water Res. 2017, 111, 66-73.

(58) Renner, R. Reaction to the Solution: Lead Exposure Following Partial Service Line Replacement. In National Institute of Environmental Health Sciences: 2010.

(59) Liu, J.; Chen, H.; Huang, Q.; Lou, L.; Hu, B.; Endalkachew, S.D.; Mallikarjuna, N.; Shan, Y.; Zhou, X. Characteristics of pipe-scale in the pipes of an urban drinking water distribution system in eastern China. Water Sci. Technol.: Water Supply 2016, 16 (3), 715-726.

(60) Chemloul, N. S.; Chaib, K.; Mostefa, K. Simultaneous measurements of the solid particle's velocity and concentration profiles in two phase flow by pulsed ultrasonic doppler velocimetry. Journal of the Brazilian Society of Mechanical Sciences and Engineering 2009, 31 (4), 333-343.

(61) Yunus, A. C.; Cimbala, J. M. Fluid mechanics fundamentals and applications. International Edition, McGraw Hill Publication 2006, 185201.

(62) Van Thienen, P.; Vreeburg, J.; Blokker, E. Radial transport processes as a precursor to particle deposition in drinking water distribution systems. Water Res. 2011, 45 (4), 1807-1817.

(63) Chen, L.; Ling, F.; Bakker, G.; Liu, W.-T.; Medema, G.; van der Meer, W.; Liu, G. Assessing the transition effects in a drinking water distribution system caused by changing supply water quality: an indirect approach by characterizing suspended solids. Water Res. 2020, 168, 115159 . 